\title{
La comunicación y la interacción como aspectos clave de los entornos personales de aprendizaje: Perspectiva de estudiantes costarricenses de educación ${ }^{1}$
}

\author{
Communication and Interaction as Key Aspects of Personal Learning Environments: \\ Perspectives of Costa Rican Education Students ${ }^{2}$
}

\section{A Comunicação e a interação como aspectos-chave do ambiente pessoal de aprendizagem: perspectiva de estudantes costa-riquenhos de educação ${ }^{3}$}

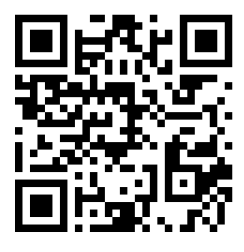

Corregido • Revised • Revisado: 19 / 06 / 2020

Aceptado • Accepted • Aprovado: 17 / 08 / 2020

\footnotetext{
${ }^{1}$ Este artículo se ha elaborado en el marco del proyecto de investigación titulado: “Ecologías de aprendizaje en la era digital: Nuevas oportunidades para la formación del profesorado de educación secundaria" (ECO4LEARN-SE), parcialmente financiado por el Ministerio de Ciencia, Innovación y Universidades (Referencia RTI2018-095690-B-I00).

2 This paper was written as part of the research proyect "Ecologías de aprendizaje en la era digital: nuevas oportunidades para la formación del profesorado de educación secundaria" (ECO4LEARN-SE), it was partially funded by Ministerio de Ciencia, Innovación y Universidades (Reference RTI2018-095690-B-I00).

${ }^{3}$ Este artigo foi preparado no âmbito do projeto de pesquisa intitulado: "Ecologías de aprendizaje en la era digital: nuevas oportunidades para la formación del profesorado de educación secundaria" (ECO4LEARN-SE), parcialmente financiado pelo Ministerio de Ciencia, Innovación y Universidades (Referência RTI2018-095690-B-I00).
} 
http://doi.org/10.15359/ree.24-3.5

http://www.una.ac.cr/educare

educare@una.ac.cr

Resumen: Gracias a la proliferación de nuevas tecnologías de la información y la comunicación, y a partir de un acceso a internet cada vez más global, se están propiciando cambios significativos tanto en la forma de trabajar en las aulas como en las relaciones que se generan entre la comunidad educativa, lo cual reclama un mayor desarrollo de las competencias comunicativas como componentes clave de los entornos personales de aprendizaje (PLE). El objetivo de esta investigación es analizar las herramientas que conoce y utiliza el estudiantado universitario de educación costarricenses, así como las actividades y mecanismos que generan con su uso para favorecer los procesos comunicativos a distintos niveles. Desde un enfoque cuantitativo, y con un diseño no experimental, se recolecta información de una muestra de 421 estudiantes a través de un cuestionario compuesto por diferentes escalas. Las principales herramientas utilizadas para compartir información son las redes sociales, el correo electrónico y las aplicaciones móviles; se desconocen, en gran medida, otros recursos. Las principales actividades se vinculan al envío de correos, utilización del chat y uso del sistema de gestión de aprendizaje (LMS). La gran mayoría de estudiantes ( $41,7 \%)$ indica que usa las herramientas tanto para compartir información como para aprender de otras personas, aunque cabe resaltar un alto porcentaje (20\%) que dice no utilizarlas para ninguna de las opciones mencionadas. Es necesario y urgente estimular y enriquecer los PLE del estudiantado para que pueda sacar mayor provecho de los recursos a su alcance para facilitar sus aprendizajes y desarrollar sus competencias comunicativas.

Palabras claves: Entorno personal de aprendizaje; tecnologías de la información y la comunicación; estudiantado universitario; competencias comunicativas; educación superior.

Abstract: Thanks to the proliferation of new information and communication technologies, and through increasingly global internet access, significant changes are being made in both the way we work in the classroom and in the relationships generated by the educational community; this calls for further development of communicative skills as key components of personal learning environments (PLE). This research aims to analyze the tools that Costa Rican education students know and use; it also examines the activities and mechanisms the students generate with their use to promote the communication processes to different levels. From a quantitative approach, and under a non-experimental design, information from a sample of 421 students is collected through a questionnaire composed of different scales. The main tools used to share information are social networks, email, and mobile apps; other resources are largely unknown. The main activities are linked to sending emails, communicating through chat applications, and using the Learning Management System (LMS). The vast majority of students (41.7\%) indicate that they use the tools to share information and learn from others; although, it is worth highlighting that a high percentage (20\%) does not use them for any of the options mentioned. It is necessary and urgent to stimulate and enrich students' PLEs so that they can make the most of the resources available to facilitate their learning and develop their communication skills.

Keywords: Personal learning environments; information technology and communication; university students; communicative competences; higher education.

Resumo: Graças à proliferação de novas Tecnologias da Informação e Comunicação, e a partir do acesso à Internet cada vez mais global, estão sendo promovidas mudanças significativas tanto na forma de trabalhar nas salas de aula quanto nas relações geradas entre a comunidade educativa, exigindo um maior desenvolvimento das competências comunicativas como componente-chave dos ambientes pessoais de aprendizagem (PLE). O objetivo desta pesquisa é analisar as ferramentas que os estudantes universitários da Costa Rica conhecem e usam, bem como as atividades e mecanismos 


\begin{abstract}
que eles geram com seu uso para favorecer processos comunicativos em diferentes níveis. A partir de uma abordagem quantitativa, e sob um desenho não experimental, as informações foram coletadas de uma amostra de 421 alunos através de um questionário composto por diferentes escalas. As principais ferramentas usadas para partilhar informação são as redes sociais, o correio eletrônico e os aplicativos móveis, desconhecendo-se, amplamente, outros recursos. As principais atividades estão relacionadas ao envio de e-mails, uso do chat e do sistema de gestão de aprendizagem (LMS). A grande maioria dos estudantes $(41,7 \%)$ indica que utiliza essas ferramentas para compartilhar informações e aprender com outras pessoas, embora valha a pena destacar um alto percentual (20\%) que diz que não as utiliza para nenhuma das opções mencionadas. É necessário e urgente estimular e enriquecer o PLE dos alunos para que possam aproveitar ao máximo os recursos à sua disposição para facilitar o aprendizado e desenvolver suas competências comunicativas.
\end{abstract}

Palavras-chave: Ambiente pessoal de aprendizagem; Tecnologias da Informação e Comunicação; estudantes universitários; competências comunicativas; educação superior.

\title{
Introducción
}

En los últimos años han proliferado las herramientas en línea que permiten la búsqueda y tratamiento de la información, facilitan su difusión y propician múltiples intercambios. Así, bajo el paraguas de la denominada web 2.0 (O'Reilly, 2005) se agrupan diversas herramientas desde las que se amplían y expanden las posibilidades de comunicación disponibles en la sociedad en red (Gutiérrez-Porlán, et al., 2018). Hace una década destacaban el correo electrónico, el chat, los SMS, el blog y la wiki, como principales recursos para la comunicación en línea (Erstad, 2006-2016), actualmente son variadas las herramientas disponibles para compartir la información y poder comunicarse, donde cobran especial relevancia las aplicaciones móviles (Figueras-Maz, et al., 2018).

En la educación superior y, específicamente, en la formación de docentes, la incorporación de las tecnologías de la información y la comunicación (TIC) está generando cambios significativos tanto en las tareas que se desarrollan en las aulas como en los mecanismos de interacción entre la comunidad educativa; cambios que influyen, de manera significativa, en las competencias comunicativas que deben poseer y saber utilizar los diferentes agentes intervinientes en el proceso de enseñanza y aprendizaje, en especial el personal docente y el estudiantado (González-Sanmamed et al., 2019; Romero-Martín, et al., 2017).

En relación con la utilización de recursos por parte del colectivo de estudiantes de educación, algunas investigaciones señalan la gran cantidad y diversidad de herramientas usadas para comunicarse y publicar, especialmente redes sociales, canales de video y aplicaciones móviles (Leiva-Núñez et al., 2018); las primeras son las preferidas para establecer contactos entre compañeros y compañeras (Gutiérrez-Porlán et al., 2018). Igualmente, Boza Carreño y Conde Vélez (2015) indican que los recursos más conocidos son aquellos que permiten la interacción social y la comunicación. Sin embargo, otros trabajos hacen énfasis en la moderada competencia del colectivo estudiantil en el uso de las TIC para fines educativos en general y de comunicación en particular (García Martínez et al., 2016; Humanate-Ramos et al., 2018). 
http://doi.org/10.15359/ree.24-3.5

http://www.una.ac.cr/educare

educare@una.ac.cr

Aunado a los cambios mencionados, hay que tener en cuenta que el binomio TIC y universidad también genera avances en otros espacios de educación informales y no formales (González-Sanmamed et al., 2018; Sangrà y Wheeler, 2013); se nutre, en ocasiones, de nuevas tendencias pedagógicas como cursos masivos y en línea (MOOC), aula invertida (flipped classroom), aprendizaje ubicuo (ULearning), entornos personales de aprendizaje (Castellanos Sánchez et al., 2017), entre otros, que se caracterizan por favorecer un aprendizaje distribuido y darle un mayor protagonismo al propio estudiantado. Algunas de estas nuevas propuestas tecnopedagógicas requieren no solo del dominio de las TIC -aspecto que según algunas investigaciones resulta familiar al estudiantado universitario (Romero-Martín et al., 2017)-, sino sobre todo el desarrollo de las competencias comunicativas, que deben trabajarse en profundidad (Gutiérrez-Porlán et al., 2018) para asegurar que el estudiantado conectado, además de dominar las TIC, asume una identidad digital de aprendiz consciente (Castellanos Sánchez et al., 2017; Roig Vila y Pascual Luna, 2012).

Entre los informes referidos al papel, las funciones y requisitos de las universidades en la era digital (Becker et al., 2017), ocupa un lugar destacado la necesidad de formar al estudiantado para el desarrollo de las competencias comunicativas, donde las TIC pueden jugar un papel relevante al propiciar el acceso a diferentes tipos de comunicación, y permitir el trabajo colaborativo y flexible en entornos tanto sincrónicos como asincrónicos y, así, mejorar el desarrollo de las habilidades sociales y emocionales (Becker et al., 2017). Esta adquisición de competencias digitales de forma genérica, y específicamente de comunicación, debe ser significativa y transferible a diversos escenarios de manera que el estudiantado esté preparado para desenvolverse en la actual sociedad del conocimiento. Es decir, no centrarse en el mero dominio de herramientas, sino hacer énfasis en aspectos relacionados con la elaboración, publicación y análisis crítico del contenido en línea (Tejada Fernández y Pozos Pérez, 2018).

Para la alfabetización digital del estudiantado universitario es recomendable tanto el conocimiento de las herramientas existentes (Becker et al., 2017), aspecto que puede derivar en un desafío, por el ritmo vertiginoso de aparición de estas mismas (Torres Kompen y Costa, 2013); así como el desarrollo de habilidades para la selección de aquellos recursos que van a formar parte de los denominados entornos personales de aprendizaje (en adelante PLE), entendidos como"el conjunto de herramientas, fuentes de información, conexiones y actividades que cada persona utiliza de forma asidua para aprender" (Adell Segura y Castañeda Quintero, 2010, p. 23).

Los PLE incluyen diferentes recursos, tecnológicos o no, aunque se nutren en mayor medida de las aplicaciones y herramientas que conforman la web 2.0 (García-Martínez y GonzálezSanmamed, 2017) y, para su óptimo desarrollo, se asocian a determinadas metodologías pedagógicas (Leiva-Núñezet al., 2018). Los PLE se configuran a través de diferentes componentes; uno de ellos corresponde a las herramientas y estrategias para relacionarse, gracias a las cuales el estudiantado puede compartir información, comunicarse y aprender con otros sujetos usuarios (Castañeda Quintero y Adell Segura, 2011), lo cual favorece el intercambio de experiencias y, 
http://doi.org/10.15359/ree.24-3.5

especialmente, el desarrollo de estrategias colaborativas (Marín-Díaz et al., 2014) y, para ello, se necesitan y, a la vez, se promueven las competencias de comunicación.

Sin embargo, la construcción y el desarrollo de los PLE no están exentos de barreras (Torres Kompen y Costa, 2013). Desde que se acuñara el término de sujetos nativos digitales, han sido múltiples las investigaciones al respecto. Los hallazgos con estudiantado universitario muestran cómo la población de menor edad ha adquirido ciertas habilidades para el uso de determinadas herramientas tecnológicas (Romero-Martín et al.,2017), especialmente de las redes sociales (Leiva-Núñez et al., 2018); sin embargo, otros trabajos advierten que su uso no está relacionado con aspectos pedagógicos o académicos (García Martínez et al., 2016; GarcíaMartínez y González-Sanmamed, 2019; Humanate-Ramos et al. 2018). Otra barrera radica en la brecha digital que se produce por determinados factores, objeto de estudio, como el nivel socioeconómico, acceso a internet, capital humano, entre otros (Matamala e Hinostroza, 2020).

Los PLE quedan vinculados a la autorregulación del aprendizaje y a la formación a lo largo de la vida (Adell Segura y Castañeda Quintero, 2010; Leiva-Núñez et al., 2018), aspecto que cobra especial interés al tratarse de profesorado en formación, ya que, como futuro sector profesional, van a jugar un papel protagónico como agente de cambio y como promotor de la formación de las nuevas generaciones. De ahí que en la formación del futuro personal docente cobre especial importancia propiciar la configuración de PLE desarrollados y enriquecidos que favorezcan la adquisición de competencias comunicativas a través de las TIC (Prendes Espinosa et al., 2018). Teniendo en cuenta lo anterior, el presente trabajo tiene el objetivo general de analizar los PLE del estudiantado universitario del último año de carrera del Centro de Investigación en Docencia y Educación (CIDE) de la Universidad Nacional de Costa Rica. Para esto se proponen los siguientes objetivos específicos:

- Determinar aquellas herramientas, finalidades y actividades que el colectivo de estudiantes utiliza y genera, en el componente de compartir información, relacionarse y aprender con otras personas.

- Identificar la existencia de diferencias significativas en el componente compartir, relacionarse y aprender con otras personas, en función de las variables edad y conexión a internet.

\section{Método}

Teniendo en cuenta los supuestos teóricos mencionados, la metodología de la investigación parte de un enfoque cuantitativo, y responde al alcance desde una doble vertiente: por un lado, exploratorio, teniendo en cuenta la falta de estudios en el ámbito costarricense que aborden los PLE de estudiantado universitario, y, por otro lado, descriptivo para dar respuesta a los objetivos planteados (Hernández Sampieri et al., 2014). En cuanto al método, y tendiendo en cuenta la no manipulación de variables, responde a un diseño no experimental y, dentro de este, transaccional, pues la recolección de datos se llevó a cabo en un solo momento (Bisquerra, 2014). 
http://doi.org/10.15359/ree.24-3.5

http://www.una.ac.cr/educare

educare@una.ac.cr

\section{Técnicas de recogida de datos}

La recogida de datos se lleva a cabo a través de la técnica de encuesta (Bisquerra, 2014), concretamente con un instrumento elaborado ad hoc, y teniendo en cuenta los constructos teóricos (buscar información, crear contenido y compartir información) que se pretenden medir a partir de la propuesta de componentes de PLE que plantean Castañeda Quintero y Adell Segura (2011).

Cabe destacar que los resultados de este artículo forman parte de una investigación de mayor envergadura, y en este caso se presentarán los datos correspondientes al quinto bloque del cuestionario, compuesto por tres escalas tipo Likert de cinco opciones de respuesta ( $1=$ nunca, $2=$ casi nunca, $3=$ algunas veces, $4=$ casi siempre y $5=$ siempre). La primera escala se refería a las herramientas (19 ítems), la segunda escala a las finalidades (10 ítems) y la última a las actividades (10 ítems) que el estudiantado utiliza para comunicarse, compartir información y aprender de otro público usuario. Previamente a estas tres escalas se les preguntaba, si conocían las diversas herramientas y solo en el caso de que la respuesta fuera afirmativa cubrían los otros aspectos que se recogían en este bloque del cuestionario.

El cuestionario fue sometido a validación de contenido por diferentes personas expertas en el área del objeto de estudio. Igualmente se realiza un pilotaje con estudiantes de características similares a la muestra definitiva. Con ambos aportes incorporados al instrumento final, se obtiene la correlación de Pearson entre los distintos componentes y la subescala total para observar el comportamiento en cuanto a la relación de las distintas variables que, en todos los casos, resultó significativa (Tabla 1).

Para el análisis de la fiabilidad, se realiza la prueba de consistencia interna alpha de Cronbach (Tabla 1). Como se observa, tanto para la subescala como para los diferentes componentes, es superior al umbral de .7, a partir del cual se pude considerar fiable (Cronbach, 1951).

Tabla 1: Matriz de correlaciones y alpha de Cronbach de la subescala y los componentes

\begin{tabular}{lccccc}
\hline & Escala Compartir & Herramientas & Actividades & Finalidades & Alpha de Cronbach \\
\hline Escala Compartir & 1 & $.882^{* *}$ & $.690^{* *}$ & $.552^{* *}$ & .896 \\
Herramientas & $.882^{* *}$ & 1 & $.551^{* *}$ & $.361^{* *}$ & .817 \\
Actividades & $.690^{* *}$ & $.551^{* *}$ & 1 & $.445^{* *}$ & .801 \\
Finalidades & $.552^{* *}$ & $.361^{* *}$ & $.445^{* *}$ & 1 & .908 \\
\hline
\end{tabular}

** La correlación es significativa al nivel 01 (bilateral).

Nota: Elaboración propia.

\section{Identificación de la muestra}

La muestra fue seleccionada de manera probabilística por estratos (Hernández Sampieri et al., 2014), y quedó formada por estudiantes de Educación del Centro de Investigación en 
http://doi.org/10.15359/ree.24-3.5

Docencia y Educación (CIDE), concretamente del último año de carrera (tanto de bachillerato licenciatura y maestría), del campus Omar Dengo de la Universidad Nacional, Costa Rica.

Para el tamaño de la muestra se utilizó la fórmula de cálculo para poblaciones finitas (Arnal et al., 1992). Teniendo en cuenta la población ( $\mathrm{N}=2333)$; un error de muestreo de $3 \%$; un nivel de confianza de $95 \%$, y la proporción esperada ( $p=5 \%$ ), se obtiene un valor de 187 para la justificación de la selección muestral. El número de instrumentos aplicados válidos asciende a $n=421$, muy por encima del dato obtenido en la aplicación de la fórmula.

Respecto a algunos estadísticos de la muestra, cabe resaltar la edad, que varía entre los 19 y los 58 años ( $M d=21 ;$ Media=24; DS=5,9). Del grupo encuestado, un 84,8 \% son mujeres (357), mientras que el 15,2\% son hombres (64). En relación con el grado, un 63,8 \% cursa bachillerato, el 32,3\% licenciatura y solamente el $3.9 \%$ una maestría.

\section{Resultados}

En primer lugar, se muestran algunos datos descriptivos en relación con los dispositivos a los que tiene acceso el colectivo de estudiantes. Se observa (Tabla 2) cómo la gran mayoría (72.2 \%) utiliza una computadora portátil; seguido de la computadora de escritorio (14.2 \%); $y$, en menor medida utilizan el Smartphone (11.2\%) y las tabletas (2.4\%). Cabe destacar que casi toda la población (96.4\%) indica tener computadora propia.

En cuanto a la conexión en el lugar de residencia durante los estudios (Tabla 2), igualmente la gran mayoría $(92.9 \%)$ indica tener conexión, mientras que el $7.1 \%$ no puede conectarse. Aunado a este aspecto, la frecuencia de conexión a internet muestra que la mayoría del estudiantado se conecta varias veces al día (79.1\%); solamente una vez al día (7.4\%); varias veces a la semana (12.1\%) y por último varias veces al mes (1.4\%). En referencia a las horas que el estudiantado pasa conectado al día, se observa también una minoría (5.2\%) que indica conectarse menos de una hora diaria; el restante se distribuye entre quienes indican conectarse de una a cinco horas (34.7\%); de seis a diez horas (27.1\%) y finalmente más de 10 horas (33.0\%).

Por último, en la Tabla 2 se muestran las frecuencias en cuanto al uso de internet en las clases por parte del personal docente universitario de educación, en las respectivas carreras. Un $12.6 \%$ indica que no se usa en ninguna clase; el $37.8 \%$ señala que se usa en menos de la mitad de las clases; en la mitad de las clases aproximadamente (15.9\%), en más de la mitad de las sesiones el $18.5 \%$ y por último solamente el $15.2 \%$ indica que se utiliza en todas las clases. Igualmente se preguntó a la muestra sobre el uso necesario de internet en los diferentes cursos de la carrera, ya sea para buscar información, reflexionar o crear nuevo contenido o para compartir información y conectarse con otras personas, al respecto, solamente el $3.3 \%$ indica que no se usa en ninguno de los cursos; el $11.4 \%$ dice usarse en menos de la mitad de las asignaturas; igualmente el 6.9 \% señala que en la mitad de los cursos; en más de la mitad de los cursos $19.5 \%$ y en todos el $58.9 \%$. 
http://doi.org/10.15359/ree.24-3.5

http://www.una.ac.cr/educare

educare@una.ac.cr

Tabla 2: Frecuencias absolutas y relativas de los dispositivos utilizados y de la conexión a internet del estudiantado

\begin{tabular}{|c|c|c|c|c|c|}
\hline $\begin{array}{l}\text { Tipo de dispositivo que más utiliza para } \\
\text { conectarse a internet }\end{array}$ & $\begin{array}{c}\text { Computadora } \\
\text { portátil } \\
304(72.2 \%)\end{array}$ & $\begin{array}{c}\text { Computadora } \\
\text { escritorio } \\
60(14.2 \%)\end{array}$ & $\begin{array}{l}\text { Tablet } \\
10(2.4 \%)\end{array}$ & \multicolumn{2}{|c|}{$\begin{array}{l}\text { Smartphone } \\
47(11.2 \%)\end{array}$} \\
\hline Posesión de computadora propia & \multicolumn{3}{|c|}{$\begin{array}{c}\text { Sí } \\
406(96.4 \%)\end{array}$} & \multicolumn{2}{|c|}{$\begin{array}{c}\text { No } \\
14(3.3 \%)\end{array}$} \\
\hline Conexión a internet en lugar de residencia & \multicolumn{3}{|c|}{$\begin{array}{c}\text { Sí } \\
391(92.9 \%)\end{array}$} & \multicolumn{2}{|c|}{$\begin{array}{c}\text { No } \\
30(7.1 \%)\end{array}$} \\
\hline Frecuencia de conexión a internet & $\begin{array}{c}\text { Una vez al día } \\
31(7.4 \%)\end{array}$ & \multicolumn{2}{|c|}{$\begin{array}{c}\text { Varias veces al día } \\
333(79.1 \%)\end{array}$} & $\begin{array}{c}\text { Varias veces a la } \\
\text { semana } 51(12.1 \%)\end{array}$ & $\begin{array}{c}\text { Varias veces al mes } \\
6(1.4 \%)\end{array}$ \\
\hline Horas de conexión al día & $\begin{array}{l}\text { Menos de } 1 \\
22(5.2 \%)\end{array}$ & \multicolumn{2}{|c|}{$\begin{array}{c}\text { De } 1 \text { a } 5 \\
146(34.7 \%)\end{array}$} & $\begin{array}{c}\text { De } 6 \text { a } 10 \\
114(27.1 \%)\end{array}$ & $\begin{array}{l}\text { Más de } 10 \\
139(33.0 \%)\end{array}$ \\
\hline $\begin{array}{l}\text { Uso de internet en las clases presenciales } \\
\text { por parte del personal docente universitario }\end{array}$ & $\begin{array}{l}\text { Ninguna } \\
53(12.6 \%)\end{array}$ & $\begin{array}{c}\text { Menos de la mitad } \\
159(37.8 \%)\end{array}$ & $\begin{array}{l}\text { La Mitad aprox. } \\
\quad 67(15.9 \%)\end{array}$ & $\begin{array}{l}\text { Más de la mitad } \\
\quad 78(18.5 \%)\end{array}$ & $\begin{array}{c}\text { Todos } \\
64(15.2 \%)\end{array}$ \\
\hline $\begin{array}{l}\text { Uso necesario de internet en los cursos } \\
\text { (búsqueda información, reflexión, etc.) }\end{array}$ & $\begin{array}{l}\text { Ninguna } \\
14(3.3 \%)\end{array}$ & $\begin{array}{c}\text { Menos de la mitad } \\
48(11.4 \%)\end{array}$ & $\begin{array}{l}\text { La Mitad aprox. } \\
\text { 29(6.9\%) }\end{array}$ & $\begin{array}{l}\text { Más de la mitad } \\
\quad 82(19.5 \%)\end{array}$ & $\begin{array}{c}\text { Todos } \\
248(58.9 \%)\end{array}$ \\
\hline
\end{tabular}

Nota: Elaboración propia.

\section{Principales herramientas tecnológicas que el estudiantado incorpora en sus PLE para compartir información y aprender de otras personas}

Es similar el porcentaje de estudiantes que utiliza las herramientas tanto para compartir la información (20.4\%), como para aprender de otras personas (17.7\%). Cabe destacar que la gran mayoría (41.7 \%) indica que la usa para ambas opciones, tanto para compartir como para aprender; sin embargo, hay que tener en cuenta el alto porcentaje (20.1\%) que indica no usarlas para ninguna de las opciones ofrecidas.

En la Figura 1, se plasman las principales herramientas que el estudiantado incorpora en sus PLE para compartir información. Indican que utilizan siempre o casi siempre: el correo electrónico (77.4 \%); las aplicaciones móviles (75.0\%); las redes sociales (70.3\%), seguido en menor medida por los canales de video (55.4\%); herramientas para almacenar e intercambiar archivos (42.1\%) y, por último, recursos para crear contenido colaborativo (33.7\%). Cabe destacar el desconocimiento que el estudiantado posee acerca de un gran número de herramientas, entre ellas los cursos masivos y abiertos en línea (siglas en inglés MOOC) donde el $87.9 \%$ no los conoce; seguido de los podcasts (85.3\%) y los marcadores sociales (83.3\%). Respecto a los microbloggings, el $41.8 \%$ no los conoce y un $36.8 \%$ los conoce, pero no los utiliza nunca o casi nunca.

Otras de las herramientas que el estudiantado sí conoce, pero no utiliza, son, por un lado, las páginas wiki, donde el $24.7 \%$ indica desconocerlas, aunado a un $42.5 \%$ que dice no utilizarlas nunca o casi nunca. Respecto a los blogs, un $15.8 \%$ no los conoce y un $48.4 \%$ no los utiliza nunca o casi nunca. Por último, cabe resaltar el caso del aula virtual, que es el sistema de gestión de aprendizaje institucional, a pesar de que solamente el $4.5 \%$ no lo conoce, un $40.5 \%$ indica no utilizarlo nunca o casi nunca, mientras el $32.1 \%$ lo utiliza algunas veces y el restante $22.6 \%$ lo utiliza siempre o casi siempre. 
http://doi.org/10.15359/ree.24-3.5

http://www.una.ac.cr/educare educare@una.ac.cr

Figura 1: Principales herramientas conocidas y usadas por el colectivo discente para compartir información

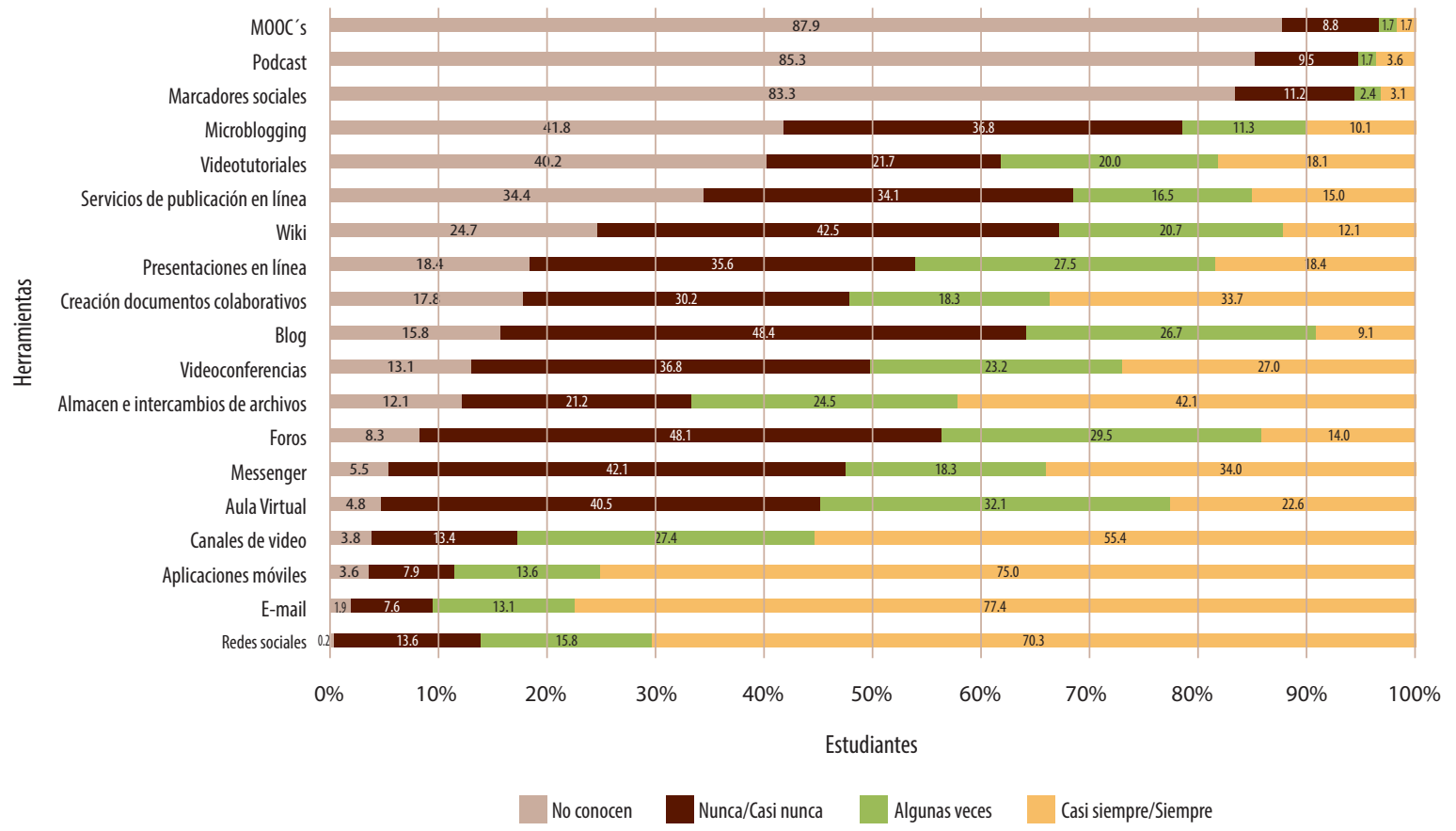

Nota: Elaboración propia.

En la Figura 2, se especifica la utilidad de las diferentes herramientas que el estudiantado conoce. Como se observa, para compartir información utilizan, en mayor medida, las redes sociales (37.7\%), el messenger (35.7\%); seguido de aplicaciones móviles (27\%); además del correo electrónico y recursos para almacenar e intercambiar archivos (26\%). Cabe destacar que los MOOC (4.4\%), las Wikis (5.5\%), videotutoriales (8.5\%) y marcadores sociales (8.8 \%) son los menos usados para compartir. En cambio, para generar aprendizaje se usa, en mayor medida, wiki (48.2 \%); seguido de marcadores sociales (42.8\%), además de los blogs (37.9\%) así como servicios de publicación en línea (36.4\%). El escaso uso que hacen de las aplicaciones móviles para este fin (2.5\%), así como de las redes sociales (3.0\%), es un elemento que debe señalarse.

Muchas de las herramientas las utilizan tanto para compartir como para aprender, entre estas cabe destacar el correo electrónico (64.5\%); aplicaciones móviles (63.5\%), así como el almacenamiento e intercambio de archivos (57.8 \%). Igualmente, hay personas que indican no utilizar los diferentes recursos para ninguna de las opciones, es decir, ni para compartir ni para aprender, entre ellas los podcasts (55.7\%), videotutoriales (50.8\%), así como el aula virtual (45.9 $\%)$, los MOOC (44.4\%) o los canales de video (32.9\%). 
http://doi.org/10.15359/ree.24-3.5

http://www.una.ac.cr/educare

educare@una.ac.cr

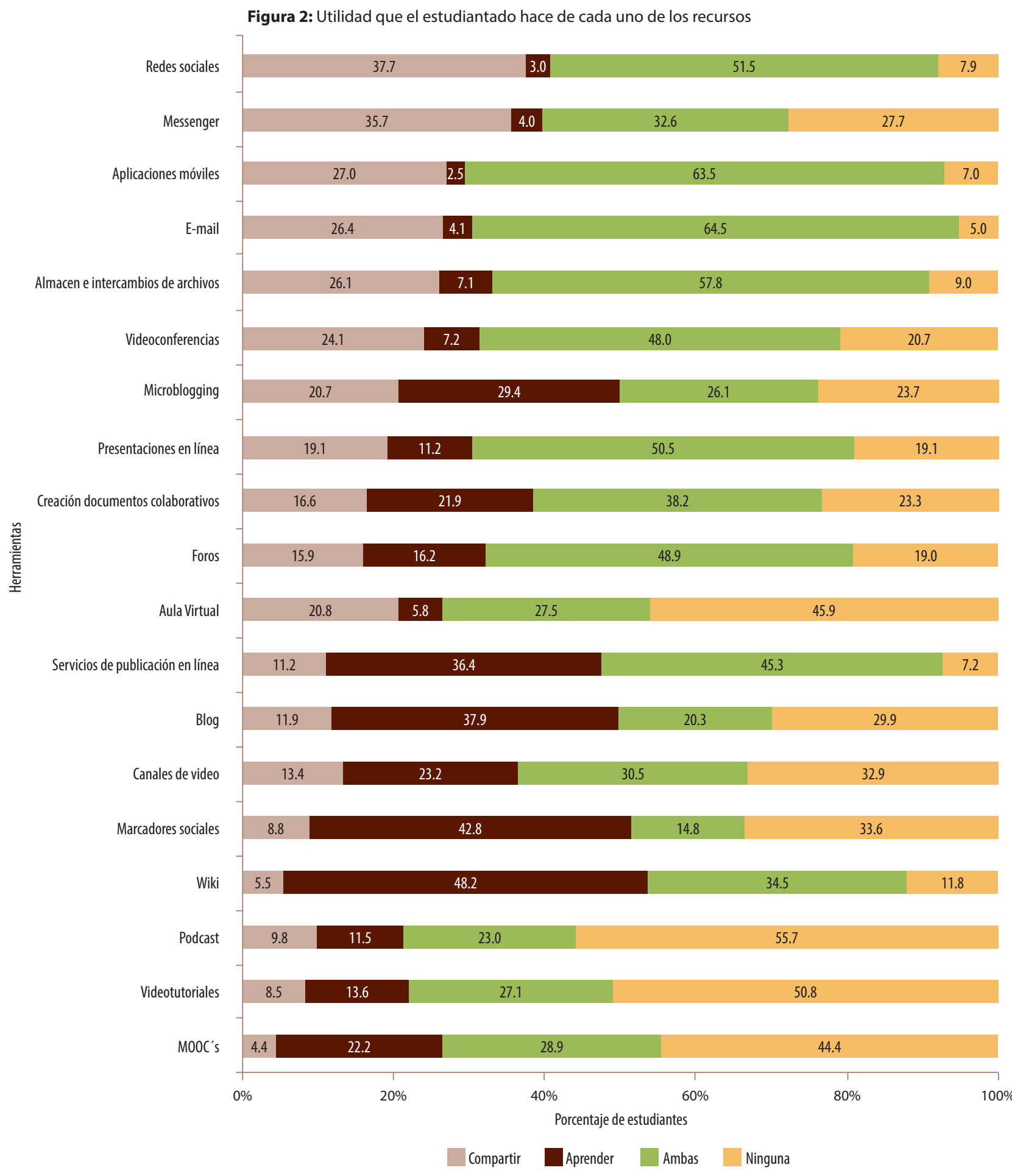

Nota: Elaboración propia. 
http://doi.org/10.15359/ree.24-3.5

http://www.una.ac.cr/educare educare@una.ac.cr

\section{Principales finalidades que motivan al estudiantado en el uso de herramientas para compartir y aprender de otras personas}

No solamente las herramientas componen los PLE, sino que también las finalidades que motivan el uso de estas. Como se observa en la Tabla 3, entre las más valoradas se encuentran el análisis de situaciones sociales ( $M=3.14, D S=0.93)$; discusión de temas de estudio $(M=3.09$, $\mathrm{DS}=0.94)$; comunicación $(\mathrm{M}=3.07, \mathrm{DS}=0.96)$, el análisis crítico $(\mathrm{M}=3.05, \mathrm{DS}=0.85)$ y el trabajo colaborativo $(\mathrm{M}=2.89, \mathrm{DS}=1.02$. Por el contrario, menos valoradas se encuentran aspectos como la capacidad de consenso $(M=2.47, D S=1.04)$, la asertividad $(M=2.51, D S=1.02)$; el liderazgo de procesos $(M=2.63, D S=1.06)$ o la resolución de conflictos $(M=2.65, D S=1.12)$.

Tabla 3: Principales finalidades que motivan al estudiantado en el uso de herramientas

\begin{tabular}{|c|c|c|c|c|c|c|c|c|}
\hline \multirow[b]{2}{*}{ Finalidades } & \multicolumn{8}{|c|}{ Frecuencias } \\
\hline & 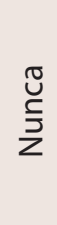 & $\begin{array}{l}\text { U } \\
\text { こ } \\
\text { ᄃ } \\
\text { U }\end{array}$ & 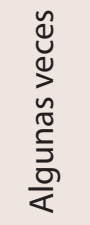 & $\begin{array}{l}\frac{0}{0} \\
\frac{0}{\varepsilon} \\
\frac{d}{n} \\
\frac{n}{U}\end{array}$ & $\frac{\frac{0}{\varrho}}{\frac{\alpha}{\tilde{U}}}$ & Total & Media & DS \\
\hline Capacidad de consenso & 30 & 39 & 144 & 141 & 67 & 421 & 2.47 & 1.04 \\
\hline Asertividad & 24 & 28 & 144 & 147 & 78 & 421 & 2.51 & 1.02 \\
\hline Liderazgo & 22 & 42 & 116 & 146 & 94 & 420 & 2.63 & 1.06 \\
\hline Resolución de conflictos & 29 & 35 & 112 & 139 & 104 & 419 & 2.65 & 1.12 \\
\hline Trabajo colaborativo & 15 & 29 & 95 & 149 & 132 & 420 & 2.89 & 1.02 \\
\hline Capacidad de decisión & 18 & 21 & 82 & 174 & 125 & 420 & 2.93 & 0.96 \\
\hline Comunicación & 15 & 21 & 70 & 155 & 160 & 421 & 3.07 & 0.96 \\
\hline Análisis crítico & 8 & 18 & 77 & 181 & 135 & 419 & 3.05 & 0.85 \\
\hline Discusión de temas de estudio & 18 & 13 & 70 & 158 & 162 & 421 & 3.09 & 0.94 \\
\hline Análisis de situaciones sociales & 15 & 15 & 65 & 153 & 173 & 421 & 3.14 & 0.93 \\
\hline
\end{tabular}

Nota: Elaboración propia.

\section{Principales actividades que genera el estudiantado en el uso de herramientas para compartir y aprender de otras personas}

Igualmente se han identificado las principales actividades que se generan con el uso de las herramientas (Tabla 4). Son utilizadas, en mayor medida, para enviar correos electrónicos $(M=3.49, D S=0.90)$, uso del chat $(M=2.97, D S=1.13)$, en el aula de clase $(M=2.36, D S=1.50)$ y a través del sistema de gestión de aprendizaje o aula virtual $(M=2.11, D S=1.24)$. En menor medida, 
http://doi.org/10.15359/ree.24-3.5

http://www.una.ac.cr/educare

educare@una.ac.cr

donde la mayoría indican no usar nunca o casi nunca estas herramientas, ha sido para congresos o seminarios en línea $(M=1.00, D S=1.03)$, mundos virtuales $(M=1.26, D S=1.31)$, tutorías virtuales $(M=1.39, D S=1.36)$, así como cursos libres y debates $(M=1.45, D S=1.18 ; M=1.52, D S=2.39)$

Tabla 4: Principales actividades que realiza el estudiantado

\begin{tabular}{|c|c|c|c|c|c|c|c|c|}
\hline \multirow[b]{2}{*}{ Actividades } & \multicolumn{8}{|c|}{ Frecuencia } \\
\hline & $\begin{array}{l}\stackrel{J}{\complement} \\
\frac{5}{Z}\end{array}$ & 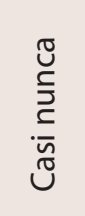 & 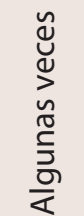 & 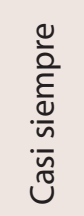 & 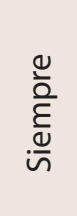 & Total & Media & DS \\
\hline $\begin{array}{l}\text { Congresos virtuales o } \\
\text { seminarios en línea }\end{array}$ & 165 & 143 & 77 & 23 & 13 & 421 & 1.00 & 1.03 \\
\hline Aula de clase & 81 & 59 & 64 & 81 & 136 & 421 & 2.36 & 1.50 \\
\hline Debates & 115 & 121 & 115 & 48 & 22 & 421 & 1.52 & 2.39 \\
\hline Cursos libres & 110 & 130 & 106 & 46 & 29 & 421 & 1.45 & 1.18 \\
\hline Mundos virtuales & 165 & 105 & 68 & 47 & 35 & 420 & 1.26 & 1.31 \\
\hline Tutorías virtuales & 158 & 92 & 67 & 65 & 38 & 420 & 1.39 & 1.36 \\
\hline Encuentros en línea & 90 & 60 & 115 & 81 & 74 & 420 & 2.02 & 1.35 \\
\hline Aula virtual & 48 & 99 & 115 & 86 & 71 & 419 & 2.11 & 1.24 \\
\hline Chat & 25 & 35 & 64 & 126 & 171 & 421 & 2.97 & 1.13 \\
\hline Correo electrónico & 15 & 13 & 33 & 78 & 282 & 421 & 3.49 & 0.90 \\
\hline
\end{tabular}

Nota: Elaboración propia.

\section{Análisis de diferencias en función de la variable edad}

En primer lugar, se realizan las pruebas de normalidad Kolmogorov-Smirnov y Shapiro-Wilk ( $p>$.05), igualmente se lleva a cabo la prueba de Levene para comprobar la homogeneidad de varianzas (0.167), para, posteriormente, generar la prueba paramétrica ANOVA de un factor, donde se han encontrado diferencias significativas en el nivel $p<.05[F(1,416)=6.9, p=.01]$ (Tabla 5). De las comparaciones post-hoc mediante la prueba HSD de Tukey (Tabla 6) se desprende que la puntuación media para el grupo de más de 30 años $(M=116.12, S D=34.4)$ fue significativas diferente del grupo de entre 19-22 años ( $M=134.38, S D=33.2)$; y del grupo de 23-26 años ( $M=138.17, S D=37.9)$. No se encontraron diferencias con respecto al grupo de entre $27-30$ años $(M=127.56, S D=40.7)$. 
Tabla 5: ANOVA. Factor de agrupación: "edad"

\begin{tabular}{lccccc} 
& Suma de cuadrados & gl & Media cuadrática & F & Sig. \\
\hline Intergrupos & 8533.724 & 1 & 8533.724 & 6.884 & .009 \\
Intragrupos & 515655.398 & 416 & 1239.556 & & \\
\hline Total & 524189.122 & 417 & & & \\
\hline
\end{tabular}

Nota: Elaboración propia.

Tabla 6: Prueba post hoc HSD de Tukey, factor "edad"

\begin{tabular}{|c|c|c|c|c|c|c|}
\hline \multirow[t]{2}{*}{ (I) Edad } & \multirow[t]{2}{*}{ (J) Edad } & \multirow[t]{2}{*}{ Diferencia de medias (I-J) } & \multirow[t]{2}{*}{ Error típico } & \multirow[t]{2}{*}{ Sig. } & \multicolumn{2}{|c|}{ Intervalo de confianza al 95\% } \\
\hline & & & & & Límite inferior & Límite superior \\
\hline \multirow{3}{*}{$\begin{array}{c}19-22 \\
\text { dimensión3 }\end{array}$} & $23-26$ & -3.782 & 4.035 & .785 & -14.19 & 6.63 \\
\hline & $27-30$ & 6.828 & 7.116 & .772 & -11.53 & 25.18 \\
\hline & Más de 30 & $18.265^{*}$ & 6.421 & .024 & 1.70 & 34.83 \\
\hline \multirow{3}{*}{$\begin{array}{c}23-26 \\
\text { dimensión3 }\end{array}$} & $19-22$ & 3.782 & 4.035 & .785 & -6.63 & 14.19 \\
\hline & $27-30$ & 10.610 & 7.548 & .497 & -8.86 & 30.08 \\
\hline & Más de 30 & $22.047^{*}$ & 6.897 & .008 & 4.26 & 39.84 \\
\hline & $19-22$ & -6.828 & 7.116 & .772 & -25.18 & 11.53 \\
\hline \multirow[t]{3}{*}{$\begin{array}{c}27-30 \\
\text { dimensión3 }\end{array}$} & $23-26$ & $-10,610$ & 7.548 & .497 & -30.08 & 8.86 \\
\hline & Más de 30 & 11.438 & 9.051 & .587 & -11.91 & 34.78 \\
\hline & $19-22$ & $-18.265^{*}$ & 6.421 & .024 & -34.83 & -1.70 \\
\hline \multirow[t]{2}{*}{$\begin{array}{c}\text { Más de } 30 \\
\text { dimensión3 }\end{array}$} & $23-26$ & $-22.047^{*}$ & $6 . .897$ & .008 & -39.84 & -4.26 \\
\hline & $27-30$ & -11.438 & 9.051 & .587 & -34.78 & 11.91 \\
\hline
\end{tabular}

* La diferencia de medias es significativa al nivel 0.05.

Nota: Elaboración propia. 
http://doi.org/10.15359/ree.24-3.5

http://www.una.ac.cr/educare

educare@una.ac.cr

\section{Análisis de diferencias en función de la variable conexión a internet}

Para comprobar diferencias entre grupos, concretamente para la variable conexión a internet en el lugar de residencia, se realiza la prueba t de Student para muestras independientes. Se obtienen diferencias significativas entre quienes indican tener conexión $(M=135.24$; $S D=$ $34.90)$ y quienes no $[M=109.31 ; S D=34.48 ; t(416)=3.86, p=, 000]$.

Igualmente se encuentran diferencias significativas en función de la frecuencia de conexión, para lo que se realizó la prueba ANOVA de un factor en el nivel $p<.05[F(3,414)=4.5$, $\mathrm{p}=.004]$. De las comparaciones post-hoc mediante la prueba HSD de Tukey se desprende que la puntuación media para el grupo que indica conectarse varias veces al día $(M=136.63, S D=35.15)$ fue significativa y diferente del grupo que indica conectarse una vez al día $(M=118.87, S D=32.60)$. No se encontraron diferencias con respecto al resto de categorías de conexión.

La misma prueba ANOVA se realiza para identificar diferencias de acuerdo con la cantidad de horas que los grupos pasan conectados diariamente, dejando entrever diferencias en el nivel $\mathrm{p}<.05[\mathrm{~F}(3,414)=8.8, \mathrm{p}=.000]$. Al igual que en el caso anterior, se genera la prueba HSD de Tukey, y se obtienen diferencias entre quienes indican estar en conexión más de 10 horas $(M=144.74$, $\mathrm{SD}=37.20)$ que arrojan diferencias significativas respecto al grupo que indica conectarse menos de una hora $(M=113.50, S D=35.52)$, entre $1-5$ horas $(M=129,64, S D=34.58)$ y quienes indican conectarse entre 6-10 horas $(M=128.41, S D=30.55)$

\section{Discusión y conclusiones}

En primer lugar, los resultados muestran cómo el estudiantado de último año de Carrera del CIDE tiene acceso a diferentes recursos tecnológicos, y la gran mayoría utiliza computadoras, tanto portátiles como de escritorio. Igualmente, un alto porcentaje posee conexión en su lugar de residencia, lo que le permite conectarse durante varias horas al día. Estos aspectos son importantes pues, de acuerdo con Torres Kompen y Costa (2013), pueden representar una barrera en la creación y desarrollo de los PLE, junto con la imposibilidad de conocer y utilizar adecuadamente todas las herramientas existentes.

Aunado a lo anterior, cabe destacar que un alto porcentaje de estudiantes indica la ausencia del uso de internet en las aulas por parte del personal docente universitario, lo que en cierta medida refleja el predominio de las clases tradicionales en las que no se están aprovechando las potencialidades de las TIC y, con ello, se reducen las posibilidades innovadoras que pueden propiciarse a partir de la incorporación de la tecnología como alternativa de los modelos tradicionales de enseñanza-aprendizaje, aspecto que supone una debilidad en la enseñanza en Costa Rica, según el Programa Estado de la Nación (2017). 
http://doi.org/10.15359/ree.24-3.5

Igualmente se observa como el alumnado universitario de educación conoce y utiliza, dentro de sus PLE, múltiples y diversas herramientas para compartir información, comunicarse o aprender con las demás personas, lo cual coincide con datos de otras investigaciones (LeivaNuñez et al., 2018). Sin embargo, hay un alto porcentaje (20 \%) que indica que su uso no está asociado a compartir ni aprender con otro público usuario, aspecto que supone una merma en los PLE y, por ende, en las competencias de comunicación del estudiantado (Gutiérrez-Porlán et al., 2018), ya que, como indica Adell Segura y Castañeda Quintero (2010), nadie aprende en aislamiento, y resalta la importancia que tiene el aprender con otras personas y de estas.

La gran mayoría de estudiantes usa el correo electrónico, aplicaciones móviles, redes sociales y canales de video, resultados que coinciden con Leiva-Nuñez et al. (2018). Pero también se detecta que desconocen, en gran parte, los MOOC, podcast y marcadores sociales, y, además, utilizan pocas herramientas como el microblogging, videotutoriales y servicios de publicación en línea, tal y como se ha señalado en otras investigaciones (Castañeda y Soto, 20l0), y donde se deja entrever la falta de aprovechamiento de la web 2.0 y la oferta de posibilidades que ofrece para la comunicación.

Son múltiples las investigaciones (Attwel, 2007; Boza Carreño y Conde Vélez, 2015; García Martínez et al., 2016; Ruiz-Palmero et al., 2013; Venkatesh et al., 2014) que indican algunas herramientas recomendables para desarrollar los PLE, entre las cuales destacan: microblogging, blog, wiki, lectores RSS, marcadores sociales, redes sociales y podcast. Los datos arrojan que gran parte de estos recursos son los más desconocidos y menos utilizados por parte del estudiantado, respecto a lo cual se coincide con Castellanos Sánchez et al. (2017), quien encontró que el estudiantado domina, en mayor medida, programas tradicionales y no las herramientas novedosas que ofrece la web 2.0. Ya se ha alertado que este aspecto supone una barrera en el desarrollo de competencias digitales $y$, por ende, en el uso crítico de las TIC, tanto a nivel personal como profesional y, especialmente, en el intercambio de información, así como en la comunicación y la participación de redes profesionales colaborativas en línea (Comisión Europea, 2007).

Se observa cómo el correo electrónico, aplicaciones móviles, el almacenamiento e intercambio de archivos, las redes sociales y las presentaciones en línea, les permiten tanto compartir como aprender de otros sujetos usuarios, elementos que cobran importancia para el desarrollo de sus PLE por la autoconsciencia del aprendizaje (Castañeda Quintero y Adell Segura, 2011) y, especialmente, en la construcción de la identidad digital de aprendizaje reflexivo (Castellanos Sánchez et al., 2017), alimentada a través de la selección crítica de herramientas eficientes. Por el contrario, se evidencia el desuso del sistema de gestión de aprendizaje (aula virtual), ni para compartir ni para aprender, en un alto porcentaje (46\%). Este hallazgo coincide con la necesidad de entornos virtuales que incorporen otros modelos de aprendizaje, además de permitir mayor agilidad y compatibilidad con prácticas metodológicas actuales (Becker et al., 2017). 
http://doi.org/10.15359/ree.24-3.5

http://www.una.ac.cr/educare

educare@una.ac.cr

Además de las herramientas, se han identificado las finalidades y actividades que el estudiantado genera dentro de sus PLE. En cuanto a las finalidades se observa que el uso de las herramientas descritas les permite el análisis de situaciones sociales, discusión de temas de estudio, comunicación, el análisis crítico y el trabajo colaborativo; aspectos que contribuyen al desarrollo de los PLE en lo personal y profesional, por la importancia que cobran las conexiones entre ambos niveles (Adell Segura y Castañeda Quintero, 2010). Cabe destacar el hecho de que utilizan las herramientas para trabajar de manera colaborativa, partiendo de la perspectiva de que el aprendizaje se deriva de una construcción social y significativa (Becker et al., 2017) que nutre el papel del estudiantado, en un futuro, como agente de transformación social.

En cuanto a las actividades que realizan, se visualiza un detrimento en los PLE, especialmente en la asistencia a congresos, seminarios en línea, mundos virtuales y cursos libres, aspecto que refleja la falta de aprovechamiento de las posibilidades que ofrece la educación informal en línea, y que coincide con uno de los retos a corto plazo de las universidades, que puede verse obstaculizado por cantidad de contenidos, la detección de su fiabilidad, así como su obsolescencia (Becker et al., 2017).

Los resultados muestran diferencias significativas en el componente herramientas, finalidades y mecanismos para compartir información, en el sentido de que aquellas personas de menor edad (rangos de 19 a 26 años) obtienen puntuaciones más elevadas que las mayores de 30, lo que implica mayor desarrollo de sus PLE en dicho componente. Este hallazgo coincide con lo planteado por Romero-Martín et al. (2017) cuando señala la facilidad que tiene el estudiantado universitario para el uso de algunas herramientas. Sin embargo, conviene tener cautela, ya que su uso no necesariamente está relacionado con aspectos académicos (García Martínez et al., 2016; Humanate-Ramos et al. 2018; Roig Vila y Pascual Luna, 2012), por lo cual es recomendable desarrollar tanto los PLE como las competencias comunicativas.

Por último, queda en evidencia la importancia que cobra actualmente el acceso a internet, ya que se ha encontrado que quienes tienen conexión durante sus estudios y señalan conectarse durante más horas obtienen puntuaciones más altas. Este aspecto muestra la necesidad de romper las brechas tecnológicas existentes (Matamala e Hinostroza, 2020), pues los resultados muestran que la conexión afecta directamente la configuración del PLE del estudiantado. A pesar de que existen múltiples recursos de aprendizaje en línea y se ha ampliado y extendido su disfrute entre toda la población, siguen existiendo dificultades de acceso para una parte de la sociedad costarricense que se presume pertenece a grupos desfavorecidos.

Los resultados alcanzados alertan sobre la necesidad de una toma de conciencia al menos a dos niveles. Por una parte, desde un punto de vista institucional, las autoridades universitarias -y particularmente las responsables de la formación del futuro personal docente- han de desarrollar las estructuras y disponer los medios para que los procesos de capacitación atiendan 
http://doi.org/10.15359/ree.24-3.5

no solo el aprendizaje de los contenidos, sino también el desarrollo de las competencias profesionales y, en particular, las competencias comunicativas que serán imprescindibles para acceder y ejecutar adecuadamente cualquier tarea y, más aún, las funciones que se espera que realice un profesor o profesora en la era digital. Pero también el estudiantado tiene que asumir la responsabilidad de su propio aprendizaje y de los mecanismos que faciliten su desarrollo profesional, tanto en los ámbitos formales como no formales y, para ello, será fundamental disponer de competencias comunicativas, que podrán ampliarse y enriquecerse a través de las posibilidades que ofrecen las TIC.

\section{Declaración de Material complementario}

Este artículo tiene disponible como material complementario:

- La versión preprint del artículo en https://doi.org/10.5281/zenodo.3765430

\section{Referencias}

Adell Segura J. y Castañeda Quintero, L. J. (2010). Los entornos personales de aprendizaje (PLEs): Una nueva manera de entender el aprendizaje. En R. Roig Vila y M. Fiorucci (Eds.), Claves para la investigación en innovación y calidad educativas. La integración de las tecnologías de la información y la comunicación y la interculturalidad en las aulas (pp. 19-30). Marfil.

Arnal, J., del Rincón, D. y Latorre, A. (1992). Investigación educativa. Fundamentos y metodología. Labor.

Attwell, G. (2007). Personal learning environments - the future of elearning? eLearning Papers, 2(1), 1-7. https://goo.gl/EYBz8e

Bisquerra, R. (Coord.). (2014). Metodología de la investigación educativa. Editorial La Muralla.

Boza Carreño, A. y Conde Vélez, S. (2015). Web 2.0 en educación superior: Formación, actitud, uso, impacto, dificultades y herramientas. Digital Education Review, 28, 45-58. http:// revistes.ub.edu/index.php/der/article/view/14423

Castañeda Quintero, L. J. y Adell Segura, J. (2011). El desarrollo profesional de los docentes en entornos personales de aprendizaje (PLE). En R. Roig-Vila y C. Laneve (Eds.), La práctica educativa en la sociedad de la información: Innovación a través de la investigación (pp. 8395). Marfil.

Castañeda, L. y Soto, J. (2010). Building personal learning environments by using and mixing ICT tools in a professional way. Digital Education Review, 18, 9-25. http://revistes.ub.edu/index. $\mathrm{php/der/article/view/11319}$ 
http://doi.org/10.15359/ree.24-3.5

http://www.una.ac.cr/educare

educare@una.ac.cr

Castellanos Sánchez, A., Sánchez Romero, C. y Calderero Hernández, J. F. (2017). Nuevos modelos tecnopedagógicos. Competencia digital de los alumnos universitarios. Revista Electrónica de Investigación Educativa, 19(1), 1-9. http://dx.doi.org/10.24320/redie.2017.19.1.1148

Comisión Europea. (2007). Competencias clave para el aprendizaje permanente. Un marco de referencia europeo. Oficina de Publicaciones Oficiales de las Comunidades Europeas. https://www.mecd.gob.es/dctm/ministerio/educacion/mecu/movilidad-europa/ competenciasclave.pdf?documentld=0901e72b80685fb1

Cronbach, L. J. (1951). Coefficiente alfa and the internal structures of tests. Psychometrika, 16(3), 297-334. https://doi.org/10.1007/BF02310555

Erstad, O. (2006-2016). Educating the digital generation. Exploring media literacy for the 21st century. Nordic Journal of Digital Literacy, 85-102. https://www.idunn.no/file/ pdf/66808577/\#page $=85$

Figueras-Maz, M., Ferrés, J., y Mateus, J.-C. (2018). Percepción de los/as coordinadores/as de la innovación docente en las universidades españolas sobre el uso de dispositivos móviles en el aula. Prisma Social, 20, 160-179. http://revistaprismasocial.es/article/view/2342

García-Martínez, J. A. y González-Sanmamed, M. (2017). Entornos personales de aprendizaje de estudiantes universitarios costarricenses de educación: Análisis de las herramientas de búsqueda de información. Revista de Investigación Educativa, 35(2), 389-407. https://doi. org/10.6018/rie.35.2.253101

García-Martínez, J. A. y González-Sanmamed, M. (2019). Cómo generan y gestionan contenidos los estudiantes de educación de Costa Rica: Una contribución al estudio de su entorno personal de aprendizaje. Digital Education Review, 36, 15-35. https://revistes.ub.edu/index. php/der/article/view/22274

García Martínez, J. A., Fallas Vargas, M. A., y Gamboa Jiménez, A. (2016). Desarrollo del entorno personal de aprendizaje: Valoración de una experiencia con estudiantes universitarios. Summa Psicológica UST, 13(2), 83-94. https://doi.org/10.18774/448x.2016.13.317

González-Sanmamed, M., Muñoz-Carril, P.-C.y Santos-Caamaño, F.-J. (2019). Key components of learning ecologies: a Delphi assessment. British Journal of Educational Technology, 50(4), 1639-1655. https://doi.org/10.1111/bjet.12805

González-Sanmamed, M., Sangrà, A., Souto-Seijo, A. y Estévez Blanco, I. (2018). Ecologías de aprendizaje en la era digital: Desafíos para la educación superior. Publicaciones, 48(1), 2545. https://doi.org/10.30827/publicaciones.v48i1.7329 
http://doi.org/10.15359/ree.24-3.5

Gutiérrez-Porlán, I., Román-García, M., y Sánchez-Vera, M. del M. (2018). Estrategias para la comunicación y el trabajo colaborativo en red de los estudiantes universitarios. Comunicar, 26(54), 91-100. https://doi.org/10.3916/C54-2018-09

Hernández Sampieri, R., Fernández Collado, C. y Baptista Lucio, P. (2014). Metodología de la investigación (6. ${ }^{a}$ ed.). McGraw-Hill.

Leiva-Núñez, J. P., Cabero-Almenara, J., y Ugalde-Meza, L. (2018). Entornos personales de aprendizaje (PLE) en estudiantes universitarios de Pedagogía. Revista Latinoamericana de Tecnología Educativa, 17(1), 25-39. http://dx.medra.org/10.17398/1695-288X.17.1.25

Matamala, C. e Hinostroza, J. E. (2020). Factores relacionados con el uso académico de Internet en educación superior. Pensamiento Educativo. Revista de Investigación Educacional Latinoamericana, 57(1), 1-19. http://dx.doi.org/10.7764/PEL.57.1.2020.7

Marín-Díaz, V., Vásquez Martínez, A. I., y McMullin, K. J. (2014). First steps towards a university social network on personal learning environments. The International Review of Research in Open and Distributed Learning, 15(3), 93-118. https://doi.org/10.19173/irrodl.v15i3.1679

Becker, A., Cummins, M., Davis, A., Freeman, A., Hall Giesinger, C. y Ananthanarayanan, V. (2017). Resumen Informe Horizon. The NMC Horizon Report: 2017 Higher Education Edition. INTEF. https://goo.gl/CtHTQF

O'Reilly, T. (2005). What Is Web 2.0. Design Patterns and Business Models for the Next Generation of Software [O'Reilly]. https://www.oreilly.com/pub/a/web2/archive/what-is-web-20. $\underline{\text { html?page }=1}$

Prendes Espinosa, M. P., Gutiérrez Porlán, I. y Martínez Sánchez, F. (2018). Competencia digital: Una necesidad del profesorado universitario en el siglo XXI. RED: Revista de Educación a Distancia, 56. http://dx.doi.org/10.6018/red/56/7

Programa Estado de la Nación. (2017). Sexto informe estado de la educación. Autor.

Humanate-Ramos, P., Silva-Castillo, J., Solís-Mazón, M. E. y Joo-Nagata, J. (2018). Las competencias TIC en los estudiantes universitarios de primer ingreso. Revista Chakiñan, 4, 124-136. https://doi.org/10.37135/chk.002.04.10

Roig Vila, R. y Pascual Luna, A. M. (2012). Las competencias digitales de los futuros docentes. Un análisis con estudiantes de magisterio de educación infantil de la Universidad de Alicante. @tic, Revista de Innovación Educativa, 9, 53-60. http://dx.doi.org/10.7203/attic.9.1958

Romero-Martín, M. R., Castejón-Oliva, F. J., López-Pastor, V.-M. y Fraile-Aranda, A. (2017). Evaluación formativa, competencias comunicativas y TIC en la formación del profesorado. Comunicar, 25(52), 73-82. https://doi.org/10.3916/C52-2017-07 
http://doi.org/10.15359/ree.24-3.5

http://www.una.ac.cr/educare

educare@una.ac.cr

Ruiz-Palmero, J., Sánchez-Rodríguez, J. y Gómez García, M. (2013). Entornos personales de aprendizaje:Estado de la situación en laFacultad deCiencias de la Educación de la Universidad de Málaga. Pixel Bit. Revista de Medios y Educación, 42, 171-181. https://goo.gl/8QEtjS

Sangrà, A. y Wheeler, S. (2013). Nuevas formas de aprendizaje informales: ¿O estamos formalizando lo informal? RUSC. Revista de Universidad y Sociedad del Conocimiento, 10(1), 107-115. https://dialnet.unirioja.es/servlet/articulo?codigo $=4615784$

Tejada Fernández, J., y Pozos Pérez, K. V. (2018). Nuevos escenarios y competencias digitales docentes: Hacia la profesionalización docente con TIC. Profesorado, Revista de Currículum y Formación del Profesorado, 22(1), 25-51. https://goo.gl/oqRqsW

Torres Kompen, R. y Costa, C. (2013). Formación continua, aprendizaje a lo largo de la vida y PLEs. En L. Castañeda y J. Adell (Eds.), Entornos personales de aprendizaje: Claves para el ecosistema educativo en red (pp. 85-92). Marfil. http://www.edutec.es/publicaciones/ entornos-personales-aprendizaje-claves-ecosistema-educativo-red

Venkatesh, V., Croteau, A.-M. y Rabah, J. (2014). Perceptions of effectiveness of instructional uses of technology in higher education in an era of Web 2.0. EN 47th Hawaii International Conference on System Science (pp. 110-119). IEEE. https://doi.org/10.1109/HICSS.2014.22 